\title{
MOLECULAR REARRANGEMENTS OF HIGHLY FUNCTIONALIZED TERPENES. AN UNIQUE REACTIVITY OF BICYCLIC FRAMEWORK AND POLIENIC CHAIN INHIBITION UNDER SUPERACIDIC TREATMENT
}

\author{
Marina Grinco, Veaceslav Kulcițki, Pavel F. Vlad, Alic Barba, \\ Elena Gorincioi, NiconUngur* \\ Institutul de Chimie al Academiei de Ştiințe a Moldovei, 3, Academiei str., Chisinau MD-2028, Republic of Moldova \\ *e-mail:nicon.ungur@gmail.com,phone/fax: (+373 22) 739775
}

\begin{abstract}
Synthesis of polyfunctional triterpene derivative [8(27),13E,17E,21E]-15-phenylsulfonyl-16-oxobicyclofarnesylfarnesol benzyl ether (8) from commercially available monoterpene geraniol and diterpene manool has been accomplished in $73 \%$ yield and its chemical transformation in superacid medium has been investigated. An unexpected rearrangement of $\mathbf{8}$ occurred, which involved methyl migration in the bicyclic fragment and total inhibition of the lateral polienic chain. A new bicyclic triterpene product [5(10),13E,17E,21E]-15-phenylsulfonyl16 -oxo-30(10 $\rightarrow 9)$-abeo-bicyclofarnesylfarnesol benzyl ether $(9)$, with rearranged new carbon skeleton has been obtained. Its bicyclic moiety is analogous to this of a natural triterpene neopolypodatetraene.
\end{abstract}

Keywords: triterpenes, synthesis, superacid, isomerization.

\section{Introduction}

Triterpenes is a group of terpenes with a high structural diversity, which includes natural products with more than 100 types of skeleton [1-3]. Reviews related to biological activities of triterpenes appear regularly and are focused on their anti-inflammatory [4], antitumor [5], anti-HIV [6,7] and insecticidal [8] activities, and also their use in treatment of metabolic and vascular diseases [9].

\section{Results and discussions}

The aim of the present work is development of the method for synthesis of bicyclic polyfunctional triterpenes with a new carbon skeletons and study of their conversion in superacid medium.

Synthesis of the bicyclic triterpenoids containing two structural blocks, fragment A (monoterpene - aliphatic) and fragment B (diterpene - bicyclic) has been drawn, in order to achieve the proposed goal.

Fragment A has been obtained from commercially available monoterpene - geraniol (1) in three steps (Scheme 1). Treatment of geraniol (1) with benzyl chloride in dichloromethane and sodium hydride led to the corresponding benzyl ether. Its spectral data and physico-chemical constants are in accordance with the reported ones [10]. Compound 2 has been subjected to oxidation with selenium dioxide in ethanol furnishing the $\alpha, \omega$-bifunctionalized derivative $\mathbf{3}$ in a modest $45 \%$ yield. Finally, the monoterpene alcohol 3 has been oxidized with pyridinium chlorochromate (PCC) into the corresponding aldehyde 4 [11] (fragment A) in a 72\% yield, its overall yield is $\sim 32 \%$.

Synthesis of fragment B has been achieved starting from commercially available manool (5). It was transformed into new diterpenic phenylsulfone 6 in a two-step sequence, according to the reported method [12]. It should be mentioned that from reaction product by column chromatography also the 13Z- izomer of diterpenyl-phenylsulfone 6 was obtained in $\sim 20 \%$ yield.

The $n$-BuLi-assisted coupling reaction of aldehyde 4 (fragment A) and diterpenylphenylsulfone 6 (fragment B) in tetrahydrofuran gave bicyclic triterpene compound 7 in a $62 \%$ yield. Compound 7 was subjected to Swern oxidation giving the [8(27),13E,17E,21E]-15-phenylsulfonyl-16-oxo-bicyclofarnesylfarnesol benzyl ether (8) in acceptable yield (73\%). It should be mentioned that an alternative oxidation of compound 7 with PCC in dichloromethane, led to a complex mixture of compounds where the content of target compound $\mathbf{8}$ does not exceed $\sim 30 \%$.

The structures of coupling reaction $\mathbf{7}$ and oxidation reaction 8 products have been established on the basis of their spectral data (see Experimental part).

Since the molecule of bicyclic triterpene derivative $\mathbf{8}$ is characterized by more than one double bond that is prone to chemical transformation in acid medium, the behavior of compound $\mathbf{8}$ in superacid medium at low temperature has been further explored [13-16]. Thus, following a previous elaboration, [8(27),13E,17E,21E]-15-phenylsulfonyl-16-oxobicyclofarnesylfarnesol benzyl ether $(\mathbf{8})$ has been treated with fluorosulfonic acid in 2-nitropropane at $-78^{\circ} \mathrm{C}[12,17]$. 


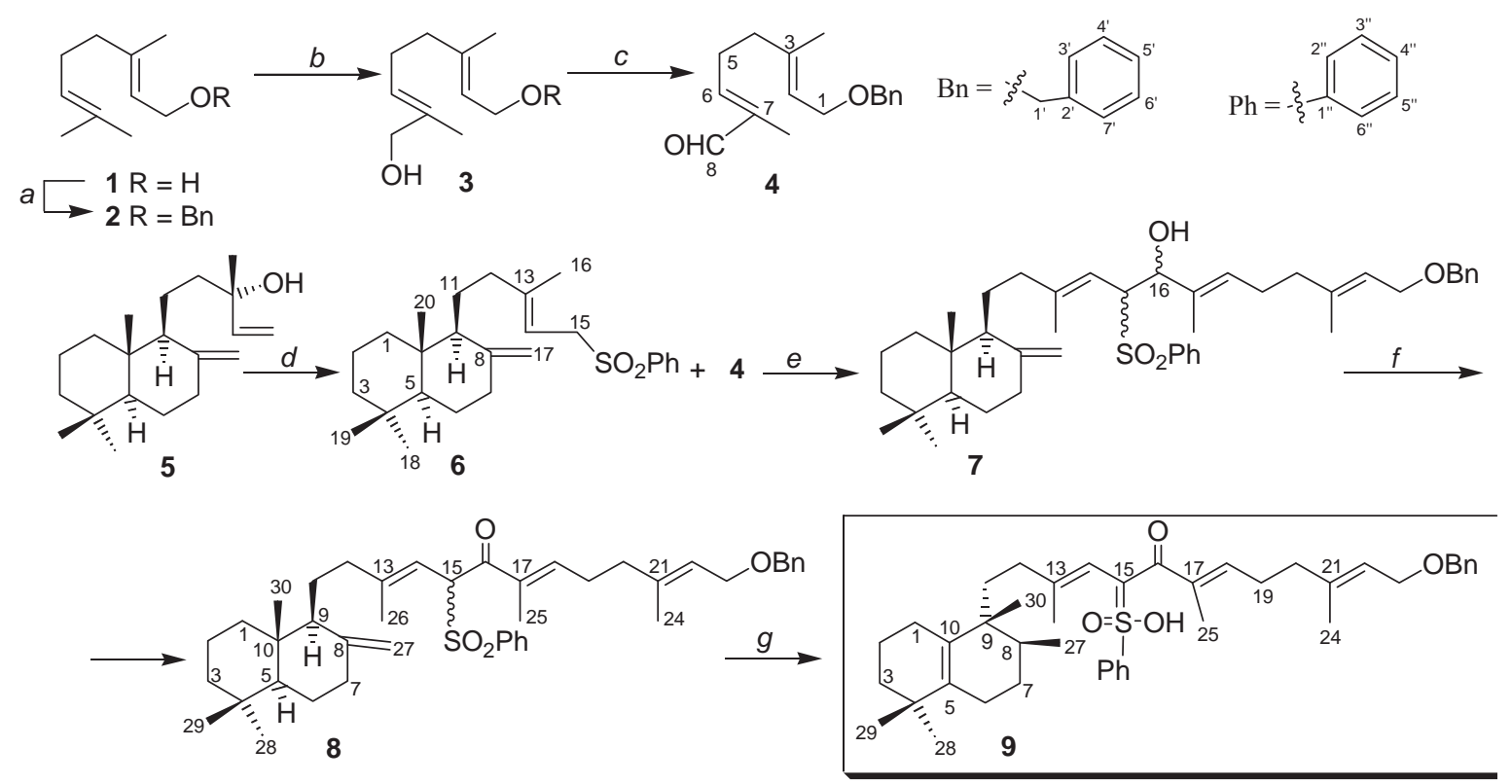

Scheme 1

Reagents and conditions. (a) $\mathrm{NaH}, \mathrm{BnCl}, \mathrm{TBAI}, \mathrm{CH}_{2} \mathrm{Cl}_{2}$, r.t., 12 h, 92\%; (b) $\mathrm{SeO}_{2}$, EtOH, reflux, 3h, 45\%; (c) PCC, $\mathrm{CH}_{2} \mathrm{Cl}_{2}$, r.t., $1.5 \mathrm{~h}, 70 \%$; (d) 1) $\left.\mathrm{PBr}_{3} / \mathrm{Et}_{2} \mathrm{O} ; 2\right) \mathrm{NaSO}_{2} \mathrm{Ph} / \mathrm{DMF}$, overall for two steps $74 \%$; $(e) n$-BuLi/THF, $66 \%$; $(f)$ $(\mathrm{COCl})_{2} / \mathrm{DMSO}, \mathrm{CH}_{2} \mathrm{Cl}_{2},-60^{\circ} \mathrm{C}, \mathrm{Et}_{3} \mathrm{~N}, 73 \%$; $(g) \mathrm{FSO}_{3} \mathrm{H}, i-\mathrm{PrNO}_{2},-78^{\circ} \mathrm{C}, 20 \mathrm{~min}, 62 \%$.

The product [5(10),13E,17E,21E]-15-phenylsulfonyl-16-oxo-30(10 $\rightarrow 9)$-abeo-bicyclofarnesylfarnesol benzyl ether (9) has been isolated in a $62 \%$ yield, its structure being assigned by IR and NMR spectroscopic data. The IR spectrum exhibited absorption bands at 1145, 1385, 1451, 1668, 1793, 2286, 2993 and $3362 \mathrm{~cm}^{-1}$, suggesting the presence of carbonyl-, phenylsulfonyl- and olefinic groups. NMR characteristics of compound $\mathbf{9}$ have been obtained on the basis of its $1 \mathrm{D}\left({ }^{1} \mathrm{H},{ }^{13} \mathrm{C}\right.$, DEPT-13 $\left.3^{\circ}\right)$ and $2 \mathrm{D}$ homo- $\left({ }^{1} \mathrm{H} /{ }^{1} \mathrm{H}\right.$ COSY- $\left.45^{\circ}\right)$ and heteronuclear $\left({ }^{1} \mathrm{H} /{ }^{13} \mathrm{C}\right.$ HSQC and ${ }^{1} \mathrm{H} /{ }^{13} \mathrm{C}$ $\mathrm{HMBC}$ ) correlation spectra (Fig. 1). The ${ }^{1} \mathrm{H}$ NMR spectrum (Table 1) displayed singlets of geminal dimethyl at $\delta_{\mathrm{H}} 0.99$, 1.01, (each $3 \mathrm{H}, \mathrm{H}-28, \mathrm{H}-29)$, signal of one tertiary and one secondary methyl groups at $\delta_{\mathrm{H}} 0.86(6 \mathrm{H}, \mathrm{H}-30, \mathrm{H}-27)$ that appears as a multiplet due to overlapping, low-field singlets of three methyls attached to double bonds at $\delta_{\mathrm{H}} 1.49,1.66$, 1.80 (each $3 \mathrm{H}, \mathrm{H}-26, \mathrm{H}-24, \mathrm{H}-25)$, downfield signals of ether methylens at $\delta_{\mathrm{H}} 4.00(d, J=6.6 \mathrm{~Hz}, \mathrm{H}-23)$ and $\delta_{\mathrm{H}} 4.50(\mathrm{~s}$, $\left.\mathrm{H}-1^{\prime}\right)$ and deshielded signals of three $\mathrm{sp}^{2}$ methines: $\delta_{\mathrm{H}} 5.44(t q, J=6.6 ; 1.1 \mathrm{~Hz}, \mathrm{H}-22), 5.74$ (br. s., H-14), 6.96 (tq, $J=$ $7.2 ; 1.2 \mathrm{~Hz}, \mathrm{H}-18$ ).

The ${ }^{1} \mathrm{H}$ NMR spectrum of compound 9 also contains the signals of two phenyl groups: one belonging to benzyl moiety, for which strong signal overlapping has been noted, at $\delta_{\mathrm{H}} 7.26-7.35\left(\mathrm{~m}, 4 \mathrm{H}, \mathrm{H}-3^{\prime}-\mathrm{-}^{\prime}\right)$ and another one, identifying phenylsulfone fragment at $\delta_{\mathrm{H}} 7.50(\mathrm{bt}, J=8.0 \mathrm{~Hz}, \mathrm{H}-3$ ", 5 " and $8.09(\mathrm{dm}, J=8.0 \mathrm{~Hz}, \mathrm{H}-2$ ", 6 "). The clear substructures H-18 - H-19 -H-20; H-14 -H-26; H-22 -H-23 - H-24 -H-20 and, in contrast to the precursor 8- H-2"- H-3", H-4", H-5"are evident in ${ }^{1} \mathrm{H} /{ }^{1} \mathrm{H}$ COSY spectrum, the accurate description of all methylene protons being difficult because of severe signal overlapping (see: Table 1). The ${ }^{13} \mathrm{C}$ NMR data exhibited thirty nine carbon signals, which were assigned by a DEPT experiment as seven methyls, eleven $\mathrm{sp}^{3}$ methylenes, one $s p^{3}$ and nine $s p^{2}$ methines, two $s p^{3}$ and ten $s p^{2}$ quaternary carbons. The presence of $\alpha, \beta$-unsaturated carbonyl moiety has been corroborated in the molecules of precursor $\mathbf{8}$, by the ${ }^{1} \mathrm{H}$ and ${ }^{13} \mathrm{C}$ NMR data $\left[\delta_{\mathrm{C}} 190.3(\mathrm{C}-16), 132.4(\mathrm{C}-17), 147.0(\mathrm{C}-18) ; \delta_{\mathrm{H}} 1.80(\mathrm{~s}, 3 \mathrm{H}, \mathrm{H}-25)\right]$. The rearranged carbon framework of 9 becomes obvious while examining its HMBC spectrum. Thus, the observed correlations from both H-6 and $\mathrm{H}-1$ to two $s p^{2}$ hybridized $\left(\mathrm{C}-5, \delta_{\mathrm{C}} 137.8\right.$ and $\left.\mathrm{C}-10, \delta_{\mathrm{C}} 131.7\right)$ instead of two sp ${ }^{3}\left(\mathrm{C}-5, \delta_{\mathrm{C}} 51.9\right.$ and $\left.\mathrm{C}-10, \delta_{\mathrm{C}} 40.4\right)$ in the former compound 8 were indicative on $\Delta^{5,10}$ localization, which was supported also by the correlations of $\mathrm{H}_{3}-29 / \mathrm{C}-5$, $\mathrm{H}_{3}-28 / \mathrm{C}-5$. The logical migration of methyl $\mathrm{H}_{3}-30$ from C-10 to C-9 position has been ascertained by $\mathrm{H}_{3}-30 / \mathrm{C}-10, \mathrm{H}_{3}-$ $30 / \mathrm{C}-9$ and $\mathrm{H}_{3}-30 / \mathrm{C}-11$ cross-peaks in the HMBC spectrum, while the evident substitution of two olefinic carbons (C-8, $\delta_{\mathrm{C}} 148.5$ and $\left.\mathrm{C}-27, \delta_{\mathrm{C}} 106.2\right)$ in the precursor 8 by the corresponding sp ${ }^{3}$ atoms in $9\left(\mathrm{C}-8, \delta_{\mathrm{C}} 33.6\right.$ and C-27, $\left.\delta_{\mathrm{C}} 16.1\right)$ has been proved by long-range correlations $\mathrm{H}_{3}-27 / \mathrm{C}-6, \mathrm{H}_{3}-27 / \mathrm{C}-7$ and $\mathrm{H}_{3}-27 / \mathrm{C}-9$. 
Table 1

\begin{tabular}{|c|c|c|c|}
\hline \multirow[t]{2}{*}{ Position } & \multicolumn{3}{|c|}{ Compound 9} \\
\hline & $\delta^{1} \mathrm{H}$ & $\mathrm{m}, J(\mathrm{~Hz})$ & $\delta^{13} \mathrm{C}^{\mathrm{a}, \mathrm{b}}$ \\
\hline 1 & $1.73,1.98$ & $\mathrm{~m}^{\mathrm{c}}$ & $25.9 \mathrm{CH}_{2}$ \\
\hline 2 & $1.49-1.65$ & $\mathrm{~m}^{\mathrm{c}}$ & $20.0 \mathrm{CH}_{2}$ \\
\hline 3 & $1.37,1.48$ & $\mathrm{~m}^{\mathrm{c}}$ & $40.0 \mathrm{CH}_{2}$ \\
\hline 4 & -- & -- & $34.6 \mathrm{qC}$ \\
\hline 5 & -- & -- & $137.8 \mathrm{qC}$ \\
\hline 6 & 1.96 & $\mathrm{~m}^{\mathrm{c}}$ & $25.2 \mathrm{CH}_{2}$ \\
\hline 7 & 1.43 & $\mathrm{~m}^{\mathrm{c}}$ & $34.2 \mathrm{CH}_{2}$ \\
\hline 8 & 1.60 & $\mathrm{~m}^{\mathrm{c}}$ & $33.6 \mathrm{qC}$ \\
\hline 9 & -- & -- & $40.6 \mathrm{qC}$ \\
\hline 10 & -- & -- & $131.7 \mathrm{qC}$ \\
\hline 11 & $1.34,1.48$ & $\mathrm{~m}^{\mathrm{c}}$ & $27.2 \mathrm{CH}_{2}$ \\
\hline 12 & $1.70,2.00$ & $\mathrm{~m}^{\mathrm{c}}$ & $35.9 \mathrm{CH}_{2}$ \\
\hline 13 & -- & -- & $150.8 \mathrm{qC}$ \\
\hline 14 & 5.74 & br. s. & $119.4 \mathrm{qC}$ \\
\hline 15 & -- & -- & not detected \\
\hline 16 & -- & -- & $190.3 \mathrm{qC}$ \\
\hline 17 & -- & -- & $132.4 \mathrm{qC}$ \\
\hline 18 & 6.96 & tq $(7.2 ; 1.2)$ & $147.0 \mathrm{CH}$ \\
\hline 19 & 2.40 & $\mathrm{~m}$ & $27.7 \mathrm{CH}_{2}$ \\
\hline 20 & 2.10 & $\mathrm{~m}$ & $37.8 \mathrm{CH}_{2}$ \\
\hline 21 & -- & -- & $138.5 \mathrm{qC}$ \\
\hline 22 & 5.44 & tq $(6.6 ; 1.1)$ & $122.0 \mathrm{CH}$ \\
\hline 23 & 4.00 & d (6.6) & $66.5 \mathrm{CH}_{2}$ \\
\hline 24 & 1.66 & $\mathrm{~s}$ & $16.5 \mathrm{CH}_{3}$ \\
\hline 25 & 1.80 & $\mathrm{~s}$ & $12.4 \mathrm{CH}_{3}$ \\
\hline 26 & 1.49 & $\mathrm{~S}$ & $18.4 \mathrm{CH}_{3}$ \\
\hline 27 & 0.86 & $\mathrm{~m}^{\mathrm{c}}$ & $16.1 \mathrm{CH}_{3}$ \\
\hline 28 & 0.99 & $\mathrm{~s}$ & $29.2 \mathrm{CH}_{3}$ \\
\hline 29 & 1.01 & $\mathrm{~s}$ & $27.7 \mathrm{CH}_{3}$ \\
\hline 30 & 0.86 & $\mathrm{~m}^{\mathrm{c}}$ & $21.2 \mathrm{CH}_{3}$ \\
\hline $1^{\prime}$ & 4.50 & $\mathrm{~s}$ & $72.3 \mathrm{CH}_{2}$ \\
\hline 2 ' & -- & -- & $138.4 \mathrm{qC}$ \\
\hline $4^{\prime}, 6^{\prime}$ & 7.30 & $\mathrm{~m}^{\mathrm{c}}$ & $127.8 \mathrm{CH}$ \\
\hline $3^{\prime}, 7^{\prime}$ & 7.30 & $\mathrm{~m}^{\mathrm{c}}$ & $128.4 \mathrm{CH}$ \\
\hline 5 , & 7.30 & $\mathrm{~m}^{\mathrm{c}}$ & $128.4 \mathrm{CH}$ \\
\hline $1 "$ & -- & -- & $136.5 \mathrm{qC}$ \\
\hline 2", 6" & 8.09 & $\mathrm{dm}(8.0)$ & $132.5 \mathrm{CH}$ \\
\hline 3", 5" & 7.50 & br.t. (8.0) & $128.2 \mathrm{CH}$ \\
\hline $4 "$ & 7.64 & $\mathrm{dm}(7.5)$ & $134.0 \mathrm{CH}$ \\
\hline
\end{tabular}

a - degree of protonation found by DEPT sequence,

b-HMBC experiments $(J=8 \mathrm{~Hz})$,

${ }^{c}-$ signal overlapping. 


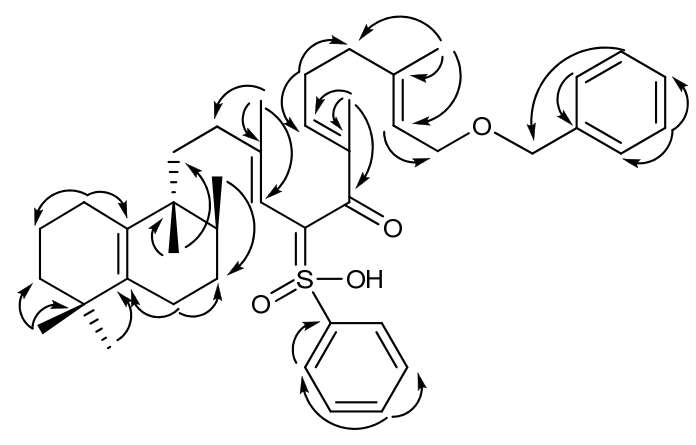

Figure 1. Selected HMBC correlations for compound 9.

The rearranged triterpene derivative $\mathbf{9}$ has been thereby obtained as a result of selective reactivity of the bicyclic part under superacidic treatment. Its plausible formation from compound $\mathbf{8}$ is depicted in Scheme 2. Due to the presence of oxygenated groups in side chain at C-16 and C-17 that can be easily protonated the bication A, which blocks the carbocyclisation in acid medium, is formed. In such a manner only the migration of proton from C-9 to C-8 takes place, producing the carbocation $\mathrm{B}$. The latter is subjected to ulterior conversion into the carbocation $\mathrm{C}$, as a result of methyl group migration from C-10 to C-9. While „quenching” the carbocation C the separation of proton at C-5 occurs and the final product 9 is obtained.

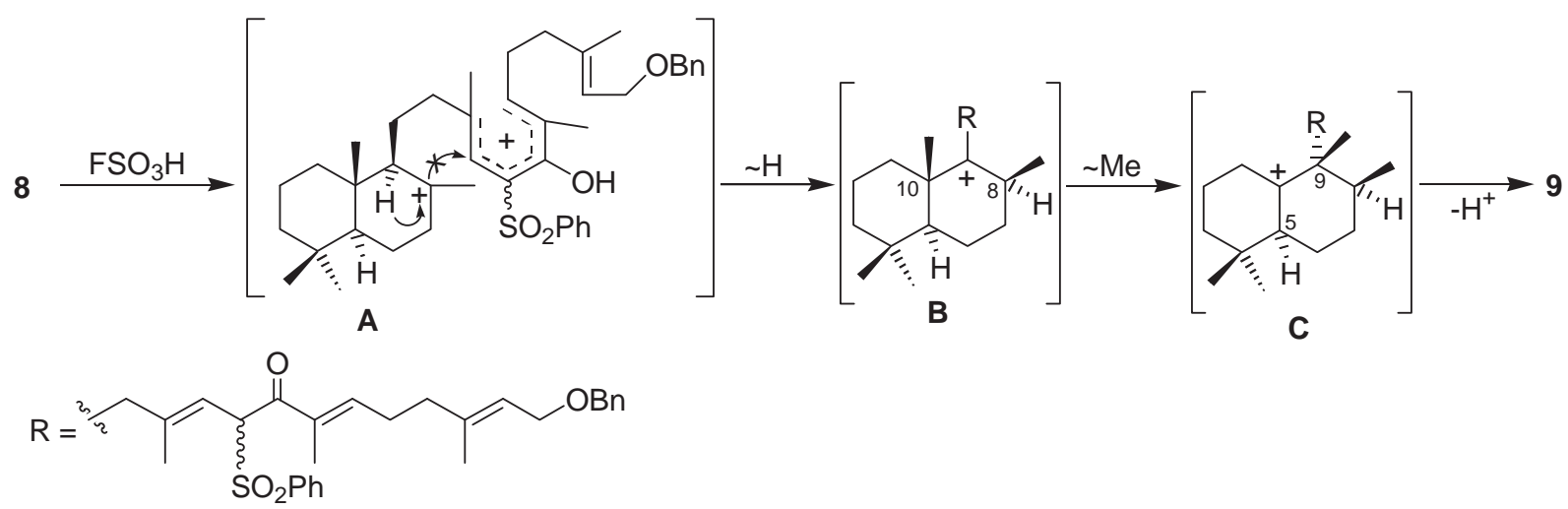

Scheme 2. Superacid-promoted molecular isomerization of compound 8.

It should be noted that the polyfunctional triterpene derivative $\mathbf{9}$ can be considered a congener of natural triterpenoid neopolypodatetraene (10), especially on considering the similarity in the bicyclic fragment (Figure 2). The latter has been isolated from a squalene hopene cyclase mutant of the prokaryotic bacterium Alicyclobacillus acidocaldarius F365A [18].
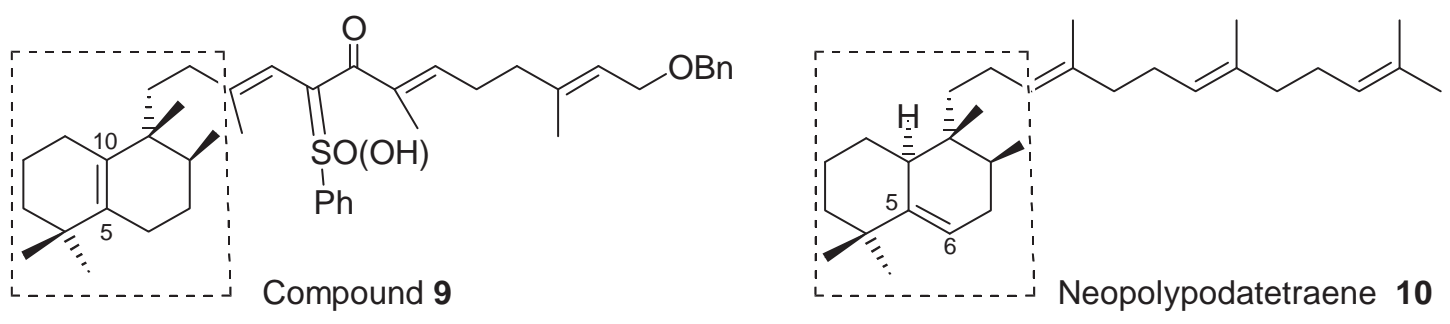

Figure 2.

\section{Conclusions}

Synthesis of polyfunctional triterpene derivative [8(27),13E,17E,21E]-15-phenylsulfonyl-16-oxobicyclofarnesylfarnesol benzyl ether has been achieved and its chemical transformation in superacid medium has been 
studied. Mentioned bicyclic triterpene product with rearranged carbon framework, which is a structural analogous of the natural triterpene neopolypodatetraene, has been obtained for the first time.

\section{Experimental}

General. Melting points (mp) were determined on a Boetius hot stage. IR Spectra were recorded on a Spectrum-100 FTIR spectrophotometer (Perkin-Elmer), with the universal ATR sampling accessory ( $\mathrm{v}$ in $\left.\mathrm{cm}^{-1}\right) .{ }^{1} \mathrm{H}-$ and ${ }^{13} \mathrm{C}-\mathrm{NMR}$ spectra were recorded in $\mathrm{CDCl}_{3}$ on Bruker 400 Avance III spectrometer $(400.13$ and $100.61 \mathrm{MHz}$ ); chemical shift are given in ppm and are referenced to measured in chloroform $\left(\mathrm{CHCl}_{3}\right)$ as internal standard $(\delta=7.26$ ppm for proton and $\delta=77.0$ for carbon). Optical rotations were measures in chloroform on a Jasco DIP 370 polarimeter, using $5 \mathrm{~cm}$ cell. Commercial Merck Si gel 60 (70-230 mesh ASTM) was used for flash chromatography, and Merck pre-coated $\mathrm{SiO}_{2}$ plates were used for TLC. The chromatograms were sprayed with $0.1 \%$ solution of cerium (IV) sulfate in $2 N$ sulfuric acid and heated at $80^{\circ} \mathrm{C}$ for $5 \mathrm{~min}$ to detect the spots. GC/MS analysis were recorded on Agilent $7890 \mathrm{~A}$ chromatograph, equipped with quadrupole MS detector MSD 5975C and HP-5 ms capillary column (30 m/0.25 mm). Treatment of reaction mixtures in organic solvents included the extraction by $\mathrm{Et}_{2} \mathrm{O}$, washing of the extract with $\mathrm{H}_{2} \mathrm{O}$ up to neutral reaction, drying over anhydrous $\mathrm{Na}_{2} \mathrm{SO}_{4}$, and solvent removal in vacuum.

Geraniol benzyl ether (2). Geraniol (1) (12 g, $77.9 \mathrm{mmol})$ in dry THF (103 mL) was treated with $60 \% \mathrm{NaH}(3.68 \mathrm{~g})$, benzyl chloride $(10.61 \mathrm{~mL}, 92.2 \mathrm{mmol})$ and tetrabutylammonium iodide $(2.88 \mathrm{~g}, 7.79 \mathrm{mmol})$. The reaction mixture was stirred for $12 \mathrm{~h}$ at room temperature. Then, $10 \%$ aqueous solution of $\mathrm{H}_{2} \mathrm{SO}_{4}(20 \mathrm{~mL})$ was added. Usual work up gave a crude reaction product ( $25 \mathrm{~g}$ ), which was purified by flash chromatography using $1 \%$ ethyl acetate/light petroleum ether mixture to yield $17.52 \mathrm{~g} \mathrm{(92 \% )} \mathrm{of} \mathrm{the} \mathrm{geraniol} \mathrm{benzyl} \mathrm{ether} \mathrm{(2),} \mathrm{as} \mathrm{a} \mathrm{pale} \mathrm{yellow} \mathrm{oil.} \mathrm{IR} \mathrm{liquid} \mathrm{film}\left(\mathrm{v}, \mathrm{cm}^{-1}\right): 735,1069$, 1101, 1270, 1377, 1453, 1496, 1670, 1726, 2924. ${ }^{1} \mathrm{H}$ NMR (400 MHz, $\left.\delta_{\mathrm{H}}\right): 1.62(s, 3 \mathrm{H}, \mathrm{H}-9), 1.66$ ( $\left.s, 3 \mathrm{H}, \mathrm{H}-10\right), 1.69$ $(d, J=0.9 \mathrm{~Hz}, 3 \mathrm{H}, \mathrm{H}-8), 2.06$ ( $m, 2 \mathrm{H}, \mathrm{H}-4), 2.12(m, 2 \mathrm{H}, \mathrm{H}-5), 4.04$ (dd, J =6.8, $0.3 \mathrm{~Hz}, 2 \mathrm{H}, \mathrm{H}-1), 4.51(s, 2 \mathrm{H}, \mathrm{H}-1$ ') $)$ $5.12(m, 1 \mathrm{H}, \mathrm{H}-6), 5.42(m, 1 \mathrm{H}, \mathrm{H}-2), 7.34(\mathrm{~m}, 5 \mathrm{H}, \mathrm{Ar}-\mathrm{H}) .{ }^{13} \mathrm{C} \mathrm{NMR}\left(100 \mathrm{MHz}, \delta_{\mathrm{c}}\right): 16.4(q, \mathrm{C}-10), 17.6(q, \mathrm{C}-9), 25.6$ $(q, \mathrm{C}-8), 26.4(t, \mathrm{C}-5), 39.6(t, \mathrm{C}-4), 66.6(t, \mathrm{C}-1), 71.9$ ( $\left.t, \mathrm{C}-1^{\prime}\right), 138.6(d, \mathrm{C}-2), 124.0(d, \mathrm{C}-6), 127.5\left(d, \mathrm{C}-5^{\prime}\right), 127.8$ $(d, \mathrm{C}-3$ ', 7') 128.3 (d, C-4', 6'), $131.6(s, \mathrm{C}-7), 138.6$ ( $s, \mathrm{C}-2$ ') $), 140.3$ ( $s, \mathrm{C}-3)$. All physical properties and spectroscopic data were identical with those reported in literature [10].

8-Hydroxygeraniol benzyl ether (3). A suspension of selenium dioxide (1.45 g, $13.08 \mathrm{mmol})$ in ethanol (9 $\mathrm{mL}) \mathrm{was}$ added to a solution of geranylbenzyl ether (2) $(6.38 \mathrm{~g}, 26.15 \mathrm{mmol})$ in ethanol $(89 \mathrm{~mL})$. The mixture was refluxed for $3 \mathrm{~h}$, cooled to $0^{\circ} \mathrm{C}$, treated with $\mathrm{NaBH}_{4}(500 \mathrm{mg}, 13.08 \mathrm{mmol})$ and stirred at the same temperature for $2 \mathrm{~h}$. After, the reaction was quenched with a $10 \%$ soln. of $\mathrm{H}_{2} \mathrm{SO}_{4}(10 \mathrm{~mL})$, and the mixture was worked up as usual. The crude product (7.5 g) was submitted to flash chromatography on silica gel $(200 \mathrm{~g})$ with increasing gradient of ethyl acetate in light petroleum ether to give starting ether (2) (3.14 g, 49\%) and 8-hydroxygeraniol benzyl ether (3) (3.05 g, 45\%), as colorless viscous oil. IR liquid film $\left(v, \mathrm{~cm}^{-1}\right): 697,736,1067,1453,1639,3429 .{ }^{1} \mathrm{H}$ NMR $\left(400 \mathrm{MHz}, \delta_{\mathrm{H}}\right): 1.65(\mathrm{~s}, 3 \mathrm{H}, \mathrm{H}-10), 1.66(s, 3 \mathrm{H}$, H-9), 2.08 ( $t, J=7.3 \mathrm{~Hz}, 2 \mathrm{H}, \mathrm{H}-4), 2.17$ ( $q, J=7.3 \mathrm{~Hz}, 2 \mathrm{H}, \mathrm{H}-5), 3.97$ ( $s, 2 \mathrm{H}, \mathrm{H}-8), 4.02$ ( $d, J=6.8 \mathrm{~Hz}, 2 \mathrm{H}, \mathrm{H}-1), 4.50(s$, 2H, H-1'), 5.39 ( $m, 2 \mathrm{H}, \mathrm{H}-2$ and H-6), 7.31 ( $m, 5 \mathrm{H}, \mathrm{Ar}-\mathrm{H}) .{ }^{13} \mathrm{C}$ NMR $\left(100 \mathrm{MHz}, \delta_{\mathrm{c}}\right): 13.6(q, \mathrm{C}-9), 16.4(q, \mathrm{C}-10), 25.8$ $(t, \mathrm{C}-5), 39.1(t, \mathrm{C}-4), 66.5(t, \mathrm{C}-1), 68.9(t, \mathrm{C}-8), 72.1\left(t, \mathrm{C}-1^{\prime}\right), 121.1(d, \mathrm{C}-2), 125.5(d, \mathrm{C}-6), 127.5(d, \mathrm{C}-5$ '), $127.8(d$, C-3', 7'), 128.3 ( $\left.d, \mathrm{C}^{\prime} 4^{\prime}, 6^{\prime}\right), 135.1(s, \mathrm{C}-3), 139.9(s, \mathrm{C}-7)$. All physical properties and spectroscopic data were identical with those reported in literature [10].

8-Oxo-geraniol benzyl ether (4). Hydroxyether (3) (1.41 g, $5.42 \mathrm{mmol})$ was dissolved in dry $\mathrm{CH}_{2} \mathrm{Cl}_{2}(83 \mathrm{~mL})$ and PCC (1.75 g, $8.13 \mathrm{mmol})$ was added. After stirring the reaction mixture at room temperature for $1.5 \mathrm{~h}$, it was diluted with diethyl ether $(60 \mathrm{~mL})$ and passed through a short silica gel pad. The crude product $(1.9 \mathrm{~g})$ was subjected to flash chromatography. Elution with 4\% ethyl acetate/light petroleum ether mixture gave $980 \mathrm{mg}(70 \%)$ of unsaturated aldehyde (4), as a pale yellow oil. IR liquid film $\left(v, \mathrm{~cm}^{-1}\right): 697,736,1069,1116,1381,1453,1686,2857,2975 .{ }^{1} \mathrm{H}$ NMR $\left(400 \mathrm{MHz}, \delta_{\mathrm{H}}\right): 1.69(s, 3 \mathrm{H}, \mathrm{H}-10), 1.76(d, J=0.8 \mathrm{~Hz}, 3 \mathrm{H}, \mathrm{H}-9), 2.23(t, J=7.6 \mathrm{~Hz}, 2 \mathrm{H}, \mathrm{H}-4), 2.5(q, J=7.6 \mathrm{~Hz}, 2 \mathrm{H}$, H-5), 4.06 ( $d, J=6.7 \mathrm{~Hz}, 2 \mathrm{H}, \mathrm{H}-1), 4.51(s, 2 \mathrm{H}, \mathrm{H}-1$ '), 5.46 (apparent $t q, J=6.7,1.2 \mathrm{~Hz}, 1 \mathrm{H}, \mathrm{H}-2), 6.47(t d, J=7.6$, $1.3 \mathrm{~Hz}, 1 \mathrm{H}, \mathrm{H}-6), 7.31$ ( $m, 5 \mathrm{H}, \mathrm{Ar}-\mathrm{H}), 9.39(s, 1 \mathrm{H}, \mathrm{H}-8) .{ }^{13} \mathrm{C} \mathrm{NMR}\left(100 \mathrm{MHz}, \delta_{\mathrm{c}}\right): 9.0(q, \mathrm{C}-9), 16.2(q, \mathrm{C}-10), 26.9(t$, C-5), $37.6(t, \mathrm{C}-4), 66.3$ ( $t, \mathrm{C}-1), 72.0$ ( $\left.t, \mathrm{C}-1^{\prime}\right), 121.9$ (d, C-2), 127.4 (d, C-5'), 127.5 (d, C-3', 7'), 128.2 (d, C-4', 6'), 138.2 ( $\left.s, \mathrm{C}-2^{\prime}\right), 138.3$ ( $\left.s, \mathrm{C}-3\right), 139.3$ ( $\left.s, \mathrm{C}-7\right), 153.5$ (d, C-6), 194.8 (d, C-8). All physical properties and spectroscopic data were identical with those reported in literature [11].

Synthesis of 8(17),13E-bicyclogeranylgeranylphenylsulfone (6). A solution of phosphorus tribromide (1.54 g, 5.69 $\mathrm{mmol})$ in dry ether $(5.0 \mathrm{~mL})$ was added dropwise to a stirred solution of manool (5) $(1.20 \mathrm{~g}, 4.14 \mathrm{mmol})$ in dry ether $(10 \mathrm{~mL})$ with cooling on an ice bath. The mixture was stirred for $2 \mathrm{~h}$ at room temperature and treated with saturated $\mathrm{NaHCO}_{3}$ solution. The ether layer was separated, washed with brine, dried, and concentrated in vacuo. The resulting 
bromide $(1.25 \mathrm{~g}, 86 \%)$ was added to a solution of the sodium salt of benzenesulfonic acid $(0.90 \mathrm{~g}, 5.45 \mathrm{mmol})$ in dry DMF $(10 \mathrm{~mL})$. The mixture was stirred at room temperature under argon in the dark for $3 \mathrm{~h}$, treated with $\mathrm{NaCl}$ solution, and extracted with ether. The extract was worked up as usual to afford a liquid product that was chromatographed on silica gel ( $42 \mathrm{~g}$ ) with gradient elution by petroleum ether: AcOEt to elute $13 E$-bicyclogeranylgeranylphenylsulfone (6) ( $1.09 \mathrm{~g}$, overall for two steps $\sim 74 \%$ ), as colorless crystals, m.p. $87-88^{\circ} \mathrm{C}$ (from hexane), $[\alpha]_{\mathrm{D}}{ }^{25}+32.2^{\circ}\left(c 1.45, \mathrm{CHCl}_{3}\right)$. IR liquid film $\left(v, \mathrm{~cm}^{-1}\right): 730,1149,1306,1447,1587,1642,2927 .{ }^{~} \mathrm{H}$ NMR $\left(400 \mathrm{MHz}, \delta_{\mathrm{H}}\right): 0.65(s, 3 \mathrm{H}, \mathrm{H}-20), 0.79(s, 3 \mathrm{H}$, $\mathrm{H}-19), 0.86(s, 3 \mathrm{H}, \mathrm{H}-18), 1.29$ ( $s, 3 \mathrm{H}, \mathrm{H}-16), 3.80(d, J=8 \mathrm{~Hz}, 2 \mathrm{H}, \mathrm{H}-15), 4.42\left(d, J=1.2 \mathrm{~Hz}, 1 \mathrm{H}, \mathrm{H}_{\mathrm{b}}-17\right), 4.80(d, J=$ $\left.1.4 \mathrm{~Hz}, 1 \mathrm{H}, \mathrm{H}_{\mathrm{a}}-17\right), 5.14(t d, J=8.0,1.3 \mathrm{~Hz}, 1 \mathrm{H}, \mathrm{H}-14), 7.68(\mathrm{~m}, 5 \mathrm{H}, \mathrm{Ph}-\mathrm{H}) \cdot{ }^{13} \mathrm{C}$ NMR $\left(100 \mathrm{MHz}, \delta_{\mathrm{c}}\right): 14.5(q, \mathrm{C}-20)$, 16.2 ( $q, \mathrm{C}-16), 19.4(t, \mathrm{C}-2), 21.7$ ( $q, \mathrm{C}-19), 21.8(t, \mathrm{C}-11), 24.4(t, \mathrm{C}-6), 33.6(s, \mathrm{C}-4), 33.6$ (q, C-18), $38.3(t, \mathrm{C}-7), 38.6$ $(t, \mathrm{C}-12), 39.1(t, \mathrm{C}-1), 39.7(s, \mathrm{C}-10), 42.1(t, \mathrm{C}-3), 55.5(d, \mathrm{C}-5), 56.1(t, \mathrm{C}-15), 56.3(d, \mathrm{C}-9), 106.2(t, \mathrm{C}-17), 110.0(d$, C-14), 128.6 (d, C-3", 5"), 128.9 (d, C-2", 6"), 133.4 (d, C-4"), 138.8 (s, C-1") 147.2 (s, C-13), 148.5 (s, C-8). Found (\%): C, 75.42; $\mathrm{H}, 9.31 . \mathrm{C}_{26} \mathrm{H}_{38} \mathrm{SO}_{2}$. Calculated (\%): C, 75.31; H, 9.24.

[8(27),13E,17E,21E]-15-phenylsulfonyl-16-hydroxy-bicyclofarnesylfarnesol benzyl ether (7). Compound 6 (529 mg, $1.28 \mathrm{mmol}, 1.2$ eq.) was co-evaporated with benzene, dried under high vacuum, and then dissolved in anhydrous THF $(4.6 \mathrm{~mL})$ and cooled to $-78{ }^{\circ} \mathrm{C}$. Then $n$-BuLi $(1.7 \mathrm{M}, 0.75 \mathrm{~mL}, 1.28 \mathrm{mmol}, 1.2$ equiv) was added drop-wise to the $8(17), 13 E$-bicyclogeranylgeranylphenylsulfone (6) solution over $2 \mathrm{~min}$. under argon atmosphere. The resulting bright yellow solution was stirred at $-78^{\circ} \mathrm{C}$ for 30 min and gradually warmed to $-40^{\circ} \mathrm{C}$ over $1 \mathrm{~h}$ and then cooled to $-78^{\circ} \mathrm{C}$. Aldehyde 4 ( $279 \mathrm{mg}, 1.07 \mathrm{mmol}, 1$ equiv) was dried under high vacuum, dissolved in THF ( $4.6 \mathrm{~mL})$, cooled to $-78^{\circ} \mathrm{C}$, and added drop-wise to the sulfone anion solution using a syringe. After $30 \mathrm{~min}$ of stirring at $-78^{\circ} \mathrm{C}$, the reaction mixture was gradually heated to $-40^{\circ} \mathrm{C}$ and sat. aq. $\mathrm{NH}_{4} \mathrm{Cl}(4 \mathrm{~mL})$ was added followed by usual workup. The residue $(900 \mathrm{mg})$ was purified by silica gel flash column chromatography (10\% ethyl acetate/light petroleum ether mixture) affording compound 7 (472 mg, 66\%), as a clear yellow oil. IR liquid film ( $\left(\mathrm{v}, \mathrm{cm}^{-1}\right): 730,1144,1230,1446,2191,2290,2947$. ${ }^{1} \mathrm{H}$ NMR $\left(400 \mathrm{MHz}, \delta_{\mathrm{H}}\right): 0.64(s, 3 \mathrm{H}, \mathrm{H}-30), 0.79(s, 3 \mathrm{H}, \mathrm{H}-29), 0.86(s, 3 \mathrm{H}, \mathrm{H}-28), 1.11(s, 3 \mathrm{H}, \mathrm{H}-26), 1.49(s, 3 \mathrm{H}$, $\mathrm{H}-25), 1.61(s, 3 \mathrm{H}, \mathrm{H}-24), 3.95(m, 1 \mathrm{H}, \mathrm{H}-15), 4.00(d, J=6.7 \mathrm{~Hz}, 2 \mathrm{H}, \mathrm{H}-23), 4.37\left(d, J=0.8 \mathrm{~Hz}, 1 \mathrm{H}, \mathrm{H}_{\mathrm{b}}-27\right), 4.49(s$, $\left.2 \mathrm{H}, \mathrm{H}-1^{\prime}\right), 4.61(d, J=9.7 \mathrm{~Hz}, 1 \mathrm{H}, \mathrm{H}-16), 4.67(m, 1 \mathrm{H}, \mathrm{H}-14), 4.80\left(d, J=0.9 \mathrm{~Hz}, 1 \mathrm{H}, \mathrm{H}_{\mathrm{a}}-27\right), 5.37(t, J=6.7 \mathrm{~Hz}, 1 \mathrm{H}$, $\mathrm{H}-22), 5.42(t, J=6.8 \mathrm{~Hz}, 1 \mathrm{H}, \mathrm{H}-18), 7.31(m, 5 \mathrm{H}, \mathrm{Ar}-\mathrm{H}), 7.68(\mathrm{~m}, 5 \mathrm{H}, \mathrm{Ph}-\mathrm{H}) .{ }^{13} \mathrm{C}$ NMR $\left(100 \mathrm{MHz}, \delta_{\mathrm{c}}\right): 10.6(q, \mathrm{C}-25)$, 14.5 ( $q$, C-30), 16.1 ( $q, \mathrm{C}-26), 16.5$ ( $q$, C-24), 19.4 ( $t, \mathrm{C}-2), 21.7$ ( $q$, C-29), 24.4 ( $t, \mathrm{C}-6), 26.0(t, \mathrm{C}-11), 29.7$ ( $t, \mathrm{C}-19)$, 33.3 ( $s, \mathrm{C}-4), 33.6$ ( $q$, C-28), $38.3(t, \mathrm{C}-7), 38.8$ ( $t, \mathrm{C}-12), 38.9(t, \mathrm{C}-20), 39.2(t, \mathrm{C}-1), 39.8(s, \mathrm{C}-10), 42.2(t, \mathrm{C}-3), 55.6$ $(d, \mathrm{C}-5), 56.5(d, \mathrm{C}-9), 66.6(t, \mathrm{C}-23), 68.6(d, \mathrm{C}-15), 72.1(t, \mathrm{C}-1$ '), 76.5 ( $d$, C-16), $106.2(t, \mathrm{C}-27), 114.0(d, \mathrm{C}-14)$, 121.3 ( $d, \mathrm{C}-22), 127.5$ ( $d$, C-5'), 127.8 ( $d$, C-3', 7'), 128.3 ( $d$, C-4', 6'), 128.8 (d, C-3", 5"), 129.4 (d, C-2", 6"), 130.2 (d, C-18), $133.4(s, \mathrm{C}-17), 133.8(d, \mathrm{C}-4 "), 137.7(s, \mathrm{C}-1 "), 138.6(s, \mathrm{C}-2$ '), $139.6(s, \mathrm{C}-21), 145.3(d, \mathrm{C}-13), 148.5(s$, C-8). Found (\%): C, 76.53; H, 8.89. $\mathrm{C}_{43} \mathrm{H}_{60} \mathrm{SO}_{4}$. Calculated (\%): C, 76.74; $\mathrm{H}, 8.99$.

[8(27),13E,17E,21E]-15-phenylsulfonyl-16-oxo-bicyclofarnesylfarnesol benzyl ether (8). A solution of DMSO (0.085 $\mathrm{mL}, 1.21 \mathrm{mmol})$ in $\mathrm{CH}_{2} \mathrm{Cl}_{2}(1.8 \mathrm{~mL})$ was added dropwise to a stirred solution of oxalyl chloride $(0.07 \mathrm{~mL}, 0.61 \mathrm{mmol})$ in $\mathrm{CH}_{2} \mathrm{Cl}_{2}(1.8 \mathrm{~mL})$ cooled to $-60{ }^{\circ} \mathrm{C}$. After $5 \mathrm{~min}$ of stirring at this temperature, a solution of compound (7) $(185 \mathrm{mg}$, $0.275 \mathrm{mmol})$ in $\mathrm{CH}_{2} \mathrm{Cl}_{2}(1.8 \mathrm{~mL})$ was added dropwise. After 30 min of stirring $\left(-60^{\circ} \mathrm{C}\right)$ triethylamine $(0.5 \mathrm{~mL}, 3.03$ $\mathrm{mmol})$ was added to the reaction mixture, and after another $15 \mathrm{~min}$ the cooling bath was removed and water $(3 \mathrm{~mL})$ was added at room temperature. After separation of the phases, the aqueous phase was extracted with $\mathrm{CH}_{2} \mathrm{Cl}_{2}(3 \times 10 \mathrm{~mL})$ and the combined organic phase was subsequently washed with a $20 \% \mathrm{H}_{2} \mathrm{SO}_{4}$, a sat. $\mathrm{NaHCO}_{3}$ solution, brine to neutral pH. Drying with $\mathrm{Na}_{2} \mathrm{SO}_{4}$ and subsequent evaporation of the solvent gave a crude reaction product, which was submitted to flash chromatography ( $8 \%$ ethyl acetate/light petroleum ether) to give polar ketone $8(134 \mathrm{mg}, 0.2 \mathrm{mmol}, 73 \%)$, as a pale yellow oil. IR liquid film $\left(v, \mathrm{~cm}^{-1}\right)$ : 745, 1144, 1309, 1394, 1449, 1665, 1791, 2289, 2986, 3365. ${ }^{1} \mathrm{H}$ NMR $\left(400 \mathrm{MHz}, \delta_{\mathrm{H}}\right)$ : 0.82 ( $s, 3 \mathrm{H}, \mathrm{H}-29), 0.87$ ( $s, 3 \mathrm{H}, \mathrm{H}-28), 0.92$ ( $s, 3 \mathrm{H}, \mathrm{H}-30), 1.65$ (s, 3H, H-26), 1.68 (s, 3H, H-24), 1.82 (s, 3H, H-25), 4.04 $(d, J=6.6 \mathrm{~Hz}, 2 \mathrm{H}, \mathrm{H}-23), 4.42\left(d, J=0.9 \mathrm{~Hz}, 1 \mathrm{H}, \mathrm{H}_{\mathrm{b}}-27\right), 4.51\left(\mathrm{~s}, 2 \mathrm{H}, \mathrm{H}-1\right.$ '), $4.82\left(d, J=1.0 \mathrm{~Hz}, 1 \mathrm{H}, \mathrm{H}_{\mathrm{a}}-27\right), 5.15(d$, $J=3.0 \mathrm{~Hz}, 1 \mathrm{H}, \mathrm{H}-14), 5.46(t, J=6.6 \mathrm{~Hz}, 1 \mathrm{H}, \mathrm{H}-22), 5.63(d, J=3.0 \mathrm{~Hz}, 1 \mathrm{H}, \mathrm{H}-15), 6.69(m, 1 \mathrm{H}, \mathrm{H}-18), 7.31(m, 5 \mathrm{H}$, Ar-H), 7.65 ( $m, 5 \mathrm{H}, \mathrm{Ph}-\mathrm{H}) .{ }^{13} \mathrm{C}$ NMR $\left(100 \mathrm{MHz}, \delta_{\mathrm{c}}\right): 11.8$ ( $\left.q, \mathrm{C}-25\right), 16.4$ (q, C-24), 17.1 (q, C-26), 26.3 ( $\left.t, \mathrm{C}-11\right), 19.3$ ( $t$, C-2), 20.1 ( $q$, C-30), 21.7 ( $q$, C-29), $24.4(t$, C-6), 27.6 ( $t, \mathrm{C}-19), 33.25$ ( $s, \mathrm{C}-4), 33.28$ ( $q, \mathrm{C}-28), 37.9$ ( $t, \mathrm{C}-20), 38.3$ $(t, \mathrm{C}-7), 39.0(t, \mathrm{C}-1), 40.39(t, \mathrm{C}-12), 40.41(s, \mathrm{C}-10), 41.8(t, \mathrm{C}-3), 51.9(d, \mathrm{C}-5), 56.3(d, \mathrm{C}-9), 66.5(t, \mathrm{C}-23), 68.7(d$, $\mathrm{C}-15), 72.3(t, \mathrm{C}-1$ '), $106.2(t, \mathrm{C}-27), 113.5(d, \mathrm{C}-14), 122.2$ ( $d, \mathrm{C}-22), 127.5\left(d, \mathrm{C}-5^{\prime}\right), 127.8\left(d, \mathrm{C}-3^{\prime}, 7^{\prime}\right), 128.3(d, \mathrm{C}-4$ ', 6'), 128.5 (d, C-3", 5"), 130.0 (d, C-2", 6"), 133.7 (d, C- 4"), 137.4 (s, C-1"), 137.8 (s, C-17), 138.4 (s, C-2'), 138.6 $(s, \mathrm{C}-21), 145.0(d, \mathrm{C}-18), 147.1(s, \mathrm{C}-13), 148.5(s, \mathrm{C}-8), 192.2(s, \mathrm{C}-16)$. Found (\%): C, 76.83; H, 8.82. $\mathrm{C}_{43} \mathrm{H}_{58} \mathrm{SO}_{4}$. Calculated (\%): C, 76.97; H, 8.71.

[5(10),13E,17E,21E]-15-phenylsulfonyl-16-oxo-30(10 $\rightarrow 9)$-abeo-bicyclofarnesylfarnesol benzyl ether (9). Compound $8(40 \mathrm{mg}, 0.06 \mathrm{mmol})$ was dissolved in 2-nitropropane $(0.7 \mathrm{~mL})$ and a solution of $\mathrm{FSO}_{3} \mathrm{H}(0.017 \mathrm{~mL}, 0.3 \mathrm{mmol}, 5$ equiv.) in 2-nitropropane $(0.2 \mathrm{~mL})$ was added under argon to the resulting solution at $-78^{\circ} \mathrm{C}$. After $20 \mathrm{~min}$ of stirring, 
the reaction mixture was quenched with a solution of triethylamine $(0.5 \mathrm{~mL})$ in light petroleum ether $(0.5 \mathrm{~mL})$, then reaction mixture was wormed to room temperature, diluted with brine $(10 \mathrm{~mL})$. After usual work-up the crude reaction product (42 mg), was submitted to flash chromatography (1\% ethyl acetate/benzene) to give compound 9 (25 $\mathrm{mg}, 62 \%)$. IR liquid film $\left(v, \mathrm{~cm}^{-1}\right)$ : 740, 1145, 1312, 1385, 1451, 1668, 1793, 2286, 2993, 3362. ${ }^{1} \mathrm{H}$ NMR (see: Table 1). ${ }^{13} \mathrm{C}$ NMR (see: Table 1). Found (\%): C, 77.12; H, 8.91. $\mathrm{C}_{43} \mathrm{H}_{58} \mathrm{SO}_{4}$. Calculated (\%): C, 76.97; H, 8.71.

\section{References}

[1] Hill, R. A.; Connolly, J. D. Nat. Prod. Rep., 2013, 30 (7), pp. 1028-1065, and previous reviews.

[2] Domingo, V.; Arteaga, J. F.; del Moral, J. F. Q.; Barrero, A. F. Nat. Prod. Rep., 2009, 26 (1), pp. 115-134.

[3] Xu, R.; Fazio, G. C.; Matsuda, S. P. Phytochemistry, 2004, 65 (3), pp. 261-291.

[4] Sporn, M. B.; Liby, K. T.; Yore, M. M.; Fu, L.; Lopchuk, J. M.; Gribble, G. W. J. Nat. Prod., 2011, 74, pp. 537545.

[5] Bishayee, A.; Ahmed, S.; Brankov, N. Perloff, M. Front. Biosci., 2011, 16, pp. 980-996.

[6] Cassels, B. K.; Asencio, M. Phytochem. Rev., 2011, 10, pp. 545-564.

[7] Kuo, R.-Y.; Qian, K.; Morris-Natschke, S. L.; Lee, K.-H. Nat. Prod. Rep., 2009, 26 (10), pp. 1321-1344.

[8] Gonzalez-Coloma, A.; Lopez-Balboa, C.; Santana, O.; Reina, M. and Fraga, B. M.; Phytochem. Rev., 2011, 10, pp. 245-260.

[9] Sheng, H.; Sun, H. Nat. Prod. Rep., 2011, 28, pp. 543-593.

[10] Altman, L. J.; Ash, L.; Stuart Marson, S. Synthesis, 1974; (2), pp. 129-131.

[11] Masaki, Y.; Hashimoto, K.; Kaji, K. Tetrahedron Lett., 1978, 19 (46), pp. 4539-4542.

[12] Kulcițki, V.; Ungur, N.; Vlad, P. F. Tetrahedron, 1998, 54 (39), pp. 11925-11934.

[13] Ungur, N.; Nguen, V. T.; Popa, N. P.; Vlad, P. F. Chem. Nat. Comp., 1993, 28 (6), pp. 561-568.

[14] Korchagina, D. V.; Gavrilyuk, O. A.; Barkhash, V. A.; Vlad, P. F.; Ungur, N. D.; Popa, N. P. Zh. Org. Khim., 1993, 29 (2), pp. 323-325.

[15] Ungur, N. ; Popa, N. P.; Kulcitki, V. N.; Vlad, P. F. Chem. Nat. Comp., 1993, 29 (5), pp. 618-621.

[16] Ungur, N.; Popa, N. P.; Vlad, P. F. Chem. Nat. Comp., 1993, 29 (5), pp. 613-617.

[17] Kulcițki, V.; Grinco, M.; Barba, A.; Ungur, N.; Vlad, P. F. Chem. Nat. Comp. 2007, 43 (3), pp. 268-273.

[18] Sato, T; Hoshino, T. Biosci., Biotechnol., Biochem., 2001, 65 (10), pp. 2233-2242. 